\title{
Clinical and Microbiological Profile of Neonatal Septicemia in A Tertiary Care Hospital in Kolkata
}

\author{
Navodeep Saha ${ }^{1}$, Mallika Sengupta ${ }^{2 *}$ (D) , Soma Sarkar ${ }^{3}$ and Manideepa Sengupta ${ }^{4}$ \\ ${ }^{1}$ Medical College, Kolkata - 700 073, West Bengal, India. ${ }^{2}$ Department of Microbiology, KPC Medical College \& \\ Hospital, Kolkata - 700 032, West Bengal, India. ${ }^{3}$ Department of Microbiology, NRS Medical College \& Hospital, \\ Kolkata - 700 014, West Bengal, India. ${ }^{4}$ Department of Microbiology, Medical College, Kolkata - 700073 , West \\ Bengal, India.
}

\begin{abstract}
Septicaemia is one of the most imminent causes of mortality among neonates in hospital. This study was undertaken to find out the clinical and bacteriological outcome of neonatal septicaemia in a tertiary care hospital in Eastern India. A cross-sectional prospective study was undertaken to investigate the causative organisms of neonatal septicaemia over a period of two months in Sick Neonatal Care Unit (SNCU) of Medical College \& Hospital, Kolkata. After obtaining ethical clearance and informed consent all relevant clinical information was collected. Blood culture was performed from all clinically suspected cases of neonatal septicaemia. After culture, identification of the isolates and antimicrobial susceptibility testing was done. During the study period, a total of 119 cases of suspected neonatal septicaemia was included which consisted of 51 (42.86\%) neonates born in the institution and 68 $\mathbf{( 5 7 . 1 4 \% )}$ neonates referred to the institution from outside. Neonates born by normal vaginal delivery were more prone to septicaemia. Highest number of cases of suspected septicaemia was seen among neonates with low birth weight (60.50\%). There were $94(78.99 \%)$ early onset sepsis and $25(21.01 \%)$ late onset sepsis cases. Among the 119 cases of suspected septicemia, 69 (57.98\%) showed growth of organisms including Candida spp. in 20, Klebsiella spp. in 18, Escherichia coli in 9, Staphylococcus aureus in 8, Enterococcus spp. in 3 and coagulase negative staphylococcus (CoNS) in 11 cases. Causative agents of neonatal septicaemia should be identified early for prevention of mortality.
\end{abstract}

Keywords: Sepsis, Pathogens, Bacteria, Neonates, Mortality, Risk factors

*Correspondence: mallikacyber@gmail.com; +919894719402

(Received: March 13, 2020; accepted: June 20, 2020)

Citation: Saha N, Sengupta M, Sarkar S, Sengupta M. Clinical and Microbiological Profile of Neonatal Septicemia in A Tertiary Care Hospital in Kolkata. J Pure Appl Microbiol. 2020;14(2):1537-1543. doi: 10.22207/JPAM.14.2.52

(c) The Author(s) 2020. Open Access. This article is distributed under the terms of the Creative Commons Attribution 4.0 International License which permits unrestricted use, sharing, distribution, and reproduction in any medium, provided you give appropriate credit to the original author(s) and the source, provide a link to the Creative Commons license, and indicate if changes were made. 


\section{INTRODUCTION}

Neonatal sepsis is a systemic infection occurring in infants who are $\leq 28$ days of age. It is an important cause of morbidity and mortality of the newborn. Early onset neonatal sepsis (EOS) has been defined based on the age of onset, with bacteremia or bacterial meningitis occurring at $\leq 72$ hours in pre-term infants hospitalized in the neonatal intensive care unit (NICU) or $<7$ days in term infants. In preterm infants, EOS is defined as occurring in the first 3 days of life and is caused by pathogens transmitted vertically from mother to infant before or during delivery. Late onset sepsis (LOS) is sepsis occurring after 72 hours in NICU infants and 7 days of life in term infants, has been variably defined as occurring up to the age of $<90$ or 120 days, and may be caused by vertically or horizontally acquired pathogens ${ }^{1}$.

The pediatric consensus definition of sepsis is SIRS in the presence of or as a result of suspected or proven infection. SIRS requires either abnormal white count (total white blood cell increased or decreased for age or $>10 \%$ immature neutrophils) or abnormal core temperature $\left(>38.5^{\circ} \mathrm{C} \text { or }<36^{\circ} \mathrm{C}\right)^{2}$. Sepsis in early infancy results in one million annual deaths worldwide, most of them occurring in developing countries ${ }^{3}$.

The signs and symptoms of neonatal sepsis are nonspecific. These include fever or hypothermia, respiratory distress including cyanosis and apnea, feeding difficulty, lethargy or irritability, hypotonia, seizures, bulging fontanel, poor perfusion, bleeding problem, abdominal distention, hepatomegaly, unexplained jaundice etc. ${ }^{4}$ Due to the overlapping nature of presentation during the first few days of life, multiple conditions including prematurity, birth asphyxia, transient tachypnea, hypoglycemia, and other physiologic disturbances may be thought as infection and treated with antibiotics as bacteremia ${ }^{5}$. Sepsis has a similar clinical presentation to other common conditions in the neonatal period. The World Health Organization (WHO) identified seven clinical signs like difficulty in feeding, convulsions, movement only when stimulated, respiratory rate $>60$ per min, severe chest indrawing and axillary temperature $>37.5^{\circ} \mathrm{C}$ or $<35.5^{\circ} \mathrm{C}$. Other authors have also included cyanosis and grunting ${ }^{6}$

This cross-sectional prospective study was undertaken to investigate the causative organisms of neonatal septicaemia and to assess their antibiotic susceptibility pattern along with the risk factors and outcome of neonatal septicaemia over a period of two months in sick neonatal care unit (SNCU) of a tertiary care hospital and medical college in eastern India.

\section{MATERIALS AND METHODS}

A cross-sectional prospective study was done for a period of two months ( $17^{\text {th }}$ May 2017 to $16^{\text {th }}$ July 2017) in sick neonatal care unit (SNCU) of Department of Paediatrics and Department of Microbiology, Medical College, Kolkata, a tertiary care hospital and medical college in eastern India. This study was done under the short term studentship of ICMR.

All newborn babies, admitted in SNCU of Medical College \& Hospital, Kolkata, whose age was less than or equal to 28 days with clinical signs and symptoms of septicemia, irrespective of whether they were born in the hospital or outside were included in the study. Infants who were more than 28 days old and those whose parents refused to give consent were excluded from the study.

The required permission to conduct the study was given by the Institutional ethics committee and informed consent was obtained from all the parents for participation in the study. A detailed history at admission and post-therapeutic health status of the newborn was taken to find out any risk factors associated with neonatal sepsis.

Blood from all clinically suspected cases of neonatal septicaemia were drawn and tested for culture by automated system in a BacT/Alert (BioMerieux Inc., France). 3-4 ml blood sample was collected from each patient and put in the paediatric blood culture bottle. In most cases, only one bottle of blood culture could be done. All culture positive samples were then sub-cultured on blood agar, chocolate agar and MacConkey agar plates along with performing a direct Gram stain. The growth of organisms was seen after 24 hours and 48 hours of incubation at $37^{\circ} \mathrm{C}$. After culture, identification of the isolates was done by using standard microbiological techniques like Gram stain and biochemical tests like indole test, methyl red test, citrate test, oxidase test, sugar utilization, $\mathrm{H}_{2} \mathrm{~S}$ production etc. and automated identification by VITEK 2 Compact (BioMerieux Inc., France) whenever necessary. Antimicrobial susceptibility 
testing was done by Kirby Bauer disc diffusion method on Mueller Hinton agar and interpretation was done by the Clinical and Laboratory Standards Institute (CLSI) version 2017 guidelines ${ }^{7}$.

All data were entered in excel spreadsheet and analyzed using SPSS $16^{\text {th }}$ version. For continuous variables having normal distribution, data was summarized using mean \pm SD. Range and median were used for all continuous variables having non-normal distribution. Chi square test was used to check the categorical variables association and $p$ value $<0.05$ was taken as significant.

\section{RESULTS}

During the study period of two months (17 $7^{\text {th }}$ May 2017 to $16^{\text {th }}$ July 2017), a total of 985 babies were born in the tertiary care centre, of which there were female 468 (47.51\%) and male 517 (52.49\%) showing a gender ratio of 905 female for every 1000 male.

During the study period, a total of 119 cases of suspected neonatal septicaemia was included which consisted of $51(42.86 \%)$ neonates born in the institution and 68 (57.14\%) neonates referred to the institution from outside. It was seen that highest number of cases $(59,49.58 \%)$ of septicaemia was suspected within 2-6 hours

Table 1. Distribution of age of new born at time of admission to SNCU with symptoms $(n=119)$

\begin{tabular}{|c|c|c|c|c|}
\hline \multicolumn{2}{|c|}{ Age at Admission } & Number & \multicolumn{2}{|c|}{ Percentage (\%) } \\
\hline \multicolumn{2}{|l|}{ 2-6 hours } & 59 & \multicolumn{2}{|c|}{49.58} \\
\hline \multicolumn{2}{|c|}{$<48$ hours } & 20 & \multicolumn{2}{|c|}{16.81} \\
\hline \multicolumn{2}{|c|}{$>48$ hours } & 40 & \multicolumn{2}{|c|}{33.61} \\
\hline \multicolumn{2}{|l|}{ Total } & 119 & \multicolumn{2}{|c|}{100} \\
\hline \multicolumn{5}{|c|}{$\begin{array}{l}\text { Table 2. Distribution of the place and mode of delivery } \\
\text { of the suspected septic neonates }(n=119)\end{array}$} \\
\hline \multirow{2}{*}{$\begin{array}{l}\text { Mode of } \\
\text { delivery }\end{array}$} & \multicolumn{3}{|c|}{ Number (\%) } & \multirow[t]{2}{*}{ Total } \\
\hline & Inborn & Out & orn & \\
\hline LUCS & 25 (21.01\%) & 281 & $3.53 \%$ & $53(44.54 \%)$ \\
\hline NVD & $24(20.17 \%)$ & 391 & $2.77 \%)$ & $63(52.94 \%)$ \\
\hline AVD & $2(1.68 \%)$ & $1(0$ & $84 \%)$ & $3(2.52 \%)$ \\
\hline Total & $51(42.86 \%)$ & 681 & $7.14 \%)$ & $119(100 \%)$ \\
\hline
\end{tabular}

LUCS = Lower Uterine Caesarean Section, NVD= Normal Vaginal Delivery, AVD= Assisted Vaginal Delivery of birth (Table 1). Out of 119 suspected cases of neonatal septicaemia, $74(62.18 \%)$ were males and $45(37.82 \%)$ were females, showing a tendency of males to be suffering more from neonatal septicaemia.

Neonates born by normal vaginal delivery (NVD) were most prone to septicaemia. Inborn neonates born by lower uterine caesarean section (LUCS) had the highest (21.01\%) suspicion of septicaemia, while in outborn neonates highest $(32.77 \%)$ suspicion of septicaemia was seen in those born by normal vaginal delivery (Table 2).

Highest number of cases of suspected septicaemia was seen among neonates with low birth weight $(60.50 \%)$. Cases of suspected neonatal sepsis were almost same among pre-term $(48.74 \%)$ and term $(49.58 \%)$ neonates (Table 3 ). Suspected septic neonates showed mainly abnormal spontaneous movements followed by abnormal body temperature, muscle tone, respiratory rate, chest indrawing, abnormal body colour and grunt in the decreasing order of the sepsis symptoms (Table 4). There were 94 (78.99\%) early onset sepsis and 25 (21.01\%) late onset sepsis cases.

Among these, 23 (19.33\%) neonates died of septicaemia within the SNCU (Table 5). The significance of different variables among survivors and non-survivors has been shown in table 6 . Decreased movements, abnormal colour, chest indrawing and grunt are significantly associated with mortality $(p<0.05)$.

Table 3. Distribution of other background characteristics of patients $(n=119)$

\begin{tabular}{lll}
\hline \multicolumn{2}{l}{ Background Characteristics } & Number (\%) \\
\hline Cried at birth & Yes & $71(59.66 \%)$ \\
& No & $48(40.34 \%)$ \\
Birth weight & Low Birth Weight & $72(60.50 \%)$ \\
& (LBW) (<2.5kg) & \\
& Normal Birth Weight & $46(38.66 \%)$ \\
& (NBW) (2.5kg-4.1kg) & \\
& Birth Over-weight & $1(0.84 \%)$ \\
Birth term & (>4.1kg) & \\
of baby & Pre-Term & $58(48.74 \%)$ \\
& Term & $59(49.58 \%)$ \\
Birth-dose & Post-Term & $2(1.68 \%)$ \\
vaccine & Given & $107(89.92 \%)$ \\
& Not Given & $12(10.08 \%)$
\end{tabular}


Table 4. Clinical profile of the patients

\begin{tabular}{llll}
\hline & Normal & Abnormal & \\
\hline Spontaneous & Alert -55 & Lethargic- 62 & Comatose-2 \\
Movements & $(46.22 \%)$ & $(52.1 \%)$ & $(1.68 \%)$ \\
Body & $36.2-37.2^{\circ} \mathrm{C}-69$ & Hypothermic-46 & Hyperthermic-4 \\
Temperature & $(57.98 \%)$ & $(38.66 \%)$ & $(3.36 \%)$ \\
Respiratory rate & $30-60 / \mathrm{min}-81$ & Low $(<30)-0$ & High $(>60)-38$ \\
& $(68.07 \%)$ & $(0 \%)$ & $(31.93 \%)$ \\
Colour & Pink-91 & Pale -14 & Cyanosis -6 \\
& $(76.47 \%)$ & $(11.77 \%)$ & $(5.04 \%)$ \\
& & Skin Mottling -1 & Icteric - 7 \\
& & $(0.84 \%)$ & $(5.88 \%)$ \\
Muscle tone & Active -81 & Limp- 34 & Increased-4 \\
& $(68.07 \%)$ & $(28.57 \%)$ & $(3.36 \%)$ \\
Chest in drawing & Absent -84 & Present - 35 & \\
& $(70.59 \%)$ & $(29.41 \%)$ & \\
Grunt & Absent- 93 & Present -26 & \\
& $(78.15 \%)$ & $(21.85 \%)$ & \\
\hline
\end{tabular}

Table 5. Distribution of the babies who died of suspected neonatal septicaemia within the SNCU $(n=23)$

\begin{tabular}{lccc}
\hline $\begin{array}{l}\text { Mode of } \\
\text { Delivery }\end{array}$ & Inborn & Outborn & Total \\
\hline LUCS & 8 & 3 & 11 \\
NVD & 6 & 6 & 12 \\
Total & 14 & 9 & 23 \\
\hline
\end{tabular}

Among the 119 cases of suspected septicemia, 69 (57.98\%) showed growth of organism. Among these 69 growth, 55 babies survived and 14 babies died $(p=0.817)$. The total 69 growth included Candida spp. in 20 (28.99\%), Klebsiella spp. in 18 (26.09\%), Escherichia coli in 9 (13.04\%), Staphylococcus aureus in 8 (11.59\%), Enterococcus spp. in $3(4.35 \%)$ and coagulase negative staphylococcus (CoNS) in 11 (15.94\%) cases. The antimicrobial susceptibility of these bacteria is given in table 7 . The coagulase negative staphylococcus isolates were not considered pathogens. Among the neonates with growth of candida, 18 survived and 2 succumbed to illness ( $p$ $=0.357)$. There were only 4 isolates of multidrug resistant (MDR) Gram negative bacilli infection in the neonates of which 3 survived and one died $(p=0.58)$. There was no significant association between culture positivity, isolation of yeast and MDR organism with mortality.

\section{DISCUSSION}

During the study period, a total of 119 cases of suspected neonatal septicemia was included of which $74(62.18 \%)$ were males and 45 $(37.82 \%)$ were females. This male preponderance is similar to the findings of an audit done on early neonatal sepsis in India by Bhat et al. ${ }^{8} \mathrm{~A}$ case of EONS or early onset neonatal sepsis was defined as an infant who had clinical signs or born to mothers with potential risk factors for infection, in whom blood culture obtained within 72 hours of life, grew a bacterial pathogen. Blood culture sample included a single sample from peripheral vein or artery. Relevant data was obtained from the unit register or neonatal case records. Of 2182 neonates screened, there were 389 (17.8\%) male babies as seen by Dutta and colleagues who found male gender as risk factor for early onset sepsis ${ }^{9}$.

Highest number of cases of suspected septicemia was seen among neonates with low birth weight $(60.50 \%)$. Cases of suspected neonatal sepsis were almost same among preterm (48.74\%) and term (49.58\%) neonates. This is similar to the findings of a study done in Brazil where it was found that $44.3 \%$ of the pre-term infants having very low birth weight developed neonatal sepsis ${ }^{10}$.

Suspected septicaemic neonates showed mainly abnormal spontaneous movements followed by abnormal body temperature, muscle tone, respiratory rate, and chest indrawing, 
Table 6. Correlation of clinical profile of the patients with outcome

\begin{tabular}{|c|c|c|c|}
\hline Abnormal Characteristics & Alive $(n=96)$ & Died $(n=23)$ & $P$ value \\
\hline $\begin{array}{l}\text { Decreased movements (lethargic / comatose) } \\
\text { Hypothermic }(<36.2 \stackrel{\circ}{ } \mathrm{C})\end{array}$ & 43 (44.79\%) & $21(91.3 \%)$ & $<0.0001$ \\
\hline / hyperthermia (>37.2으) & $41(42.7 \%)$ & $9(39.13 \%)$ & 0.754 \\
\hline High respiratory rate (>60/ $\mathrm{min})$ & $31(32.29 \%)$ & $7(30.43 \%)$ & 0.863 \\
\hline $\begin{array}{l}\text { Abnormal colour (pale/ skin mottling/ } \\
\text { cyanosis/ icteric) }\end{array}$ & $16(16.67 \%)$ & $12(52.17 \%)$ & 0.0003 \\
\hline Improper muscle tone (limp/ increased) & $34(35.42 \%)$ & $4(17.39 \%)$ & 0.095 \\
\hline Chest in drawing & $23(23.96 \%)$ & $12(52.17 \%)$ & 0.007 \\
\hline Grunt & $10(10.42 \%)$ & $16(69.56 \%)$ & $<0.0001$ \\
\hline Low birth weight $(<2.5 \mathrm{~kg})$ & $60(62.5 \%)$ & $12(52.17 \%)$ & 0.362 \\
\hline Did not cry at birth & $38(39.58 \%)$ & $10(43.48 \%)$ & 0.732 \\
\hline Pre - term & $47(48.96 \%)$ & $11(47.83 \%)$ & 0.922 \\
\hline
\end{tabular}

Note - $p$-value was determined using chi-square test

Table 7. Antimicrobial susceptibility pattern of the isolated bacteria

\begin{tabular}{llllll}
\hline Antimicrobial agents & $\begin{array}{l}\text { E.coli } \\
(\mathrm{n}=9)\end{array}$ & $\begin{array}{l}\text { Klebsiella } \\
\mathrm{spp}(\mathrm{n}=18)\end{array}$ & $\begin{array}{l}\text { S. aureus } \\
(\mathrm{n}=8)\end{array}$ & $\begin{array}{l}\text { CoNS } \\
(\mathrm{n}=11)\end{array}$ & $\begin{array}{l}\text { Enterococcus } \\
\text { spp }(\mathrm{n}=3)\end{array}$ \\
\hline Ceftriaxone & $7(77.78 \%)$ & $15(83.33 \%)$ & $\mathrm{NA}$ & $\mathrm{NA}$ & $\mathrm{NA}$ \\
Cefotaxime & $7(77.78 \%)$ & $15(83.33 \%)$ & $\mathrm{NA}$ & $\mathrm{NA}$ & $\mathrm{NA}$ \\
Cefoxitin & $\mathrm{NA}$ & $\mathrm{NA}$ & $2(25 \%)$ & $6(54.54 \%)$ & $\mathrm{NA}$ \\
Amoxycillin/ clavulanate & $3(33.33 \%)$ & $6(33.33 \%)$ & $2(25 \%)$ & $6(54.54 \%)$ & NA \\
Piperacillin/ tazobactam & $8(88.89 \%)$ & $12(66.67 \%)$ & $\mathrm{NA}$ & $\mathrm{NA}$ & $\mathrm{NA}$ \\
Imipenem & $8(88.89 \%)$ & $14(77.78 \%)$ & $\mathrm{NA}$ & $\mathrm{NA}$ & $\mathrm{NA}$ \\
Meropenem & $8(88.89 \%)$ & $14(77.78 \%)$ & $\mathrm{NA}$ & $\mathrm{NA}$ & $\mathrm{NA}$ \\
Gentamicin & $4(44.44 \%)$ & $13(72.22 \%)$ & $6(75 \%)$ & $9(81.82 \%)$ & NA \\
Amikacin & $6(66.67 \%)$ & $17(94.44 \%)$ & $\mathrm{NA}$ & $\mathrm{NA}$ & $\mathrm{NA}$ \\
Ciprofloxacin & $7(77.78 \%)$ & $14(77.78 \%)$ & $3(37.5 \%)$ & $2(18.19 \%)$ & $2(66.67 \%)$ \\
Levofloxacin & $8(88.89 \%)$ & $16(88.89 \%)$ & $3(37.5 \%)$ & $4(36.37 \%)$ & $3(100 \%)$ \\
Colistin & $9(100 \%)$ & $18(100 \%)$ & NA & NA & NA \\
Vancomycin & NA & NA & $8(100 \%)$ & $11(100 \%)$ & $3(100 \%)$ \\
Teicoplanin & NA & NA & $8(100 \%)$ & $11(100 \%)$ & $3(100 \%)$ \\
Linezolid & NA & NA & $8(100 \%)$ & $11(100 \%)$ & $3(100 \%)$ \\
\hline
\end{tabular}

NA = Not applicable

abnormal body colour and grunt in the decreasing order of the sepsis symptoms. In a study done by Tsai et $a$ it was found that late onset neonatal sepsis was associated with low birth weight and less gestational age ${ }^{11}$. The different risk factors for early-onset neonatal sepsis are prematurity, maternal Group B streptococcal colonization, prolonged rupture of membranes, and maternal intra-amniotic infection. Late-onset neonatal sepsis is attributable to Gram-positive organisms, including coagulase negative Staphylococci and Staphylococcus aureus ${ }^{12}$.
There were 94 (78.99\%) early onset sepsis and 25 (21.01\%) late onset sepsis cases. In a study done by Bizzarro et $a l$ it was found that $90 \%$ of cases were late onset sepsis. E. coli and Group B Streptococcus was the most common organism associated with early onset sepsis while for late onset sepsis the most common agents were coagulase negative staphylococci (CoNS) and Staphylococcus aureus ${ }^{13}$. In a study done by Turhan et al blood cultures were found to be positive in $47.6 \%$ patients of neonatal sepsis among 351 babies. The bacteria which cause mortality 
with the highest rate included $E$. coli, coagulase negative staphylococci, S. aureus and Klebsiella $\mathrm{sp}^{14}$. In the present study the important causative organisms were Candida spp. in 20 (28.99\%), Klebsiella spp. in 18 (26.09\%), Escherichia coli in 9 (13.04\%), Staphylococcus aureus in 8 (11.59\%), Enterococcus spp. in 3 (4.35\%) and coagulase negative staphylococcus (CoNS) in $11(15.94 \%)$ cases. Another study showed that yeasts and bacteria isolated from 235 cultures of neonatal sepsis included Enterobacteriaceae (51.5\%), followed by Streptococcus spp. in early sepsis, and by Staphylococcus spp. in late sepsis. Of all nosocomial Enterobacteriaceae, $40 \%$ were extended spectrum beta-lactamase producing bacteria. Among Staphylococcus species, resistance to oxacillin was recorded in $65.5 \%{ }^{15}$. In the present study there were $75 \%$ methicillin resistant Staphylococcus aureus (MRSA) and $45 \%$ methicillin resistant coagulase negative staphylococcus (MRCoNS). All Gram positive organisms like Staphylococcus aureus, coagulase negative staphylococcus and Enterococcus were susceptible to vancomycin, teicoplanin and linezolid.

\section{CONCLUSION}

The most important observation of this study was the fact that fungi like Candida and among bacteria, Gram-negative organisms, particularly Klebsiella and E.coli were predominant organisms causing neonatal septicaemia in this institution. The massive improvement in the quality of the medical facilities, combined with strict maintenance of hygiene, and a refined treatment protocol has succeeded in lowering mortality due to neonatal septicaemia.

\section{ACKNOWLEDGEMENTS}

None.

\section{CONFLICT OF INTEREST}

The authors declare that there is no conflict of interest.

\section{AUTHORS' CONTRIBUTION}

All authors listed above made a substantial, direct and intellectual contribution to the work and approved it for publication.

\section{FUNDING}

None.

\section{ETHICS STATEMENT}

Obtained from the Institutional Ethics Committee, Medical College Kolkata.

\section{INFORMED CONSENT}

Obtained from the parents of the neonates.

\section{DATA AVAILABILITY}

All datasets generated or analysed during the study are included in the manuscript.

\section{REFERENCES}

1. Simonsen KA, Anderson-Berry AL, Delair SF, Davies HD. Early-Onset Neonatal Sepsis. Clin Microbiol Rev. 2014;27(1):21-47. https://doi.org/10.1128/ CMR.00031-13

2. Wynn JL, Wong HR, Shanley TP, Bizzarro MJ, Saiman $L$, Polin RA. Time for a neonatal-specific consensus definition for sepsis. Pediatr Crit Care Med J Soc Crit Care Med World Fed Pediatr Intensive Crit Care Soc. 2014;15(6):523-8. https://doi.org/10.1097/ PCC.0000000000000157

3. Panigrahi P, Parida S, Nanda NC, et al. A randomized synbiotic trial to prevent sepsis among infants in rural India. Nature. 2017;548(7668):407-12. https://doi. org/10.1038/nature23480

4. Shah BA, Padbury JF. Neonatal sepsis. Virulence. 2014;5(1):170-8. https://doi.org/10.4161/viru.26906

5. Panigrahi P, Chandel DS, Hansen NI, et al. Neonatal sepsis in rural India: timing, microbiology, and antibiotic resistance in a population-based prospective study in the community setting. J Perinatol off $J$ Calif Perinat Assoc. 2017;37(8):911-21. https://doi. org/10.1038/jp.2017.67

6. Zea-Vera A, Ochoa TJ. Challenges in the diagnosis and management of neonatal sepsis. J Trop Pediatr. 2015;61(1):1-13. https://doi.org/10.1093/tropej/ fmu079

7. Clinical and Laboratory Standards Institute, editor. Performance standards for antimicrobial disk susceptibility test: twenty-fifth informational supplement. Wayne, PA: Committee for Clinical Laboratory Standards; 2015. 236 p. (Documents / Clinical and Laboratory Standards Institute).

8. Bhat Y R, Lewis LES, KE V. Bacterial isolates of earlyonset neonatal sepsis and their antibiotic susceptibility pattern between 1998 and 2004: an audit from a center in India. Ital J Pediatr. 2011;37:32. https://doi. org/10.1186/1824-7288-37-32

9. Dutta S, Reddy R, Sheikh S, Kalra J, Ray P, Narang A. Intrapartum antibiotics and risk factors for early onset sepsis. Arch Dis Child Fetal Neonatal Ed. 2010;95(2):F99-103. https://doi.org/10.1136/ adc. 2009.163220 
10. Ferreira RC, Mello RR, Silva KS. Neonatal sepsis as a risk factor for neurodevelopmental changes in preterm infants with very low birth weight. J Pediatr (Rio J). 2014;90(3):293-299. https://doi.org/10.1016/j. jped.2013.09.006

11. Tsai M-H, Chu S-M, Lee C-W, et al. Recurrent lateonset sepsis in the neonatal intensive care unit: incidence, clinical characteristics and risk factors. Clin Microbiol Infect Off Publ Eur Soc Clin Microbiol Infect Dis. 2014;20(11):0928-935. https://doi. org/10.1111/1469-0691.12661

12. Shane AL, Stoll BJ. Neonatal sepsis: progress towards improved outcomes. J Infect. 2014;68 Suppl 1:S24-32. https://doi.org/10.1016/j.jinf.2013.09.011
13. Bizzarro MJ, Shabanova V, Baltimore RS, et al. Neonatal sepsis 2004-2013: the rise and fall of coagulasenegative staphylococci. J Pediatr. 2015;166(5):11931199. https://doi.org/10.1016/j.jpeds.2015.02.009

14. Turhan EE, Gursoy T, Ovali F. Factors which affect mortality in neonatal sepsis. Turk Pediatri Arsivi. 2015;50(3):170-5. https://doi.org/10.5152/ TurkPediatriArs.2015.2627

15. Lona Reyes JC, Verdugo Robles MA, Perez Ramirez RO, Perez Molina JJ, Ascencio Esparza EP, Benitez Vazquez EA. Etiology and antimicrobial resistance patterns in early and late neonatal sepsis in a Neonatal Intensive Care Unit. Arch Argent Pediatr. 2015;113(4):317-23. https://doi.org/10.5546/aap.2015.eng.317 